\title{
Contribution to the knowledge of the Megalodontesidae, Argidae, Cimbicidae, Cephidae and Tenthredinidae (Symphyta: Hymenoptera) fauna from Bingöl and Diyarbakır provinces of Turkey
}

\author{
Emin Kaplan ${ }^{1}$, Abdullah Mart $^{2}$, Attila Haris $^{3} \&$ Erol Yildirim$^{4}$
}

\author{
${ }^{1}$ Bingöl University, Faculty of Agriculture Department of Plant Protection, 12000 Bingöl, Turkey, \\ email: eminkaplan021@gmail.com \\ ${ }^{2}$ Osmaniye Korkut Ata University, Faculty of Arts and Sciences, Department of Biology, Osmaniye, Turkey, \\ email: abdullahmartt@osmaniye.edu.tr \\ ${ }^{3}$ H-1076 Budapest, Garay street 19., Hungary, email: attilaharis@yahoo.com \\ ${ }^{4}$ Atatürk University, Faculty of Agriculture, Department of Plant Protection, 25240-Erzurum, Turkey, \\ email: yildirimerol@hotmail.com
}

\begin{abstract}
Kaplan, E., Mart, Attila Haris, A. \& Yildirim E.: Contribution to the knowledge of the Megalodontesidae, Argidae, Cimbicidae, Cephidae and Tenthredinidae (Symphyta: Hymenoptera) fauna from Bingöl and Diyarbakur provinces of Turkey.

Abstract: This study is based upon material from Megalodontesidae, Argidae, Cimbicidae, Cephidae and Tenthredinidae collected from Bingöl and Diyarbakır provinces of Turkey between 2016 and 2018. The study resulted in recording for Turkish fauna of two species from one genera of Megalodontesidae, eight species from two genera of Argidae, two species from one genera of Cimbicidae, three species from three genera of Cephidae and 23 species from six genera of Tenthredinidae. In total, 38 species belonging to 13 genera of Symphyta are listed. Among them, Macrophya postica Brullé, 1832, Tenthredo bifasciata O. F. Müller, 1766 and Tenthredo zonula Klug, 1817 have been found the most abundant and widespread species. Separately, Tenthredo (Elinora) baetica Spinola, 1843 is newly recorded from Turkey. In addition, new localities have been found for some species, which have already been reported from Turkey.
\end{abstract}

Keywords: Hymenoptera, Symphyta, Megalodontesidae, Argidae, Cimbicidae, Cephidae, Tenthredinidae, new record, Bingöl, Diyarbakır, Muş, Turkey,

\section{Introduction}

Turkey occupies Asia Minor between the Mediterranean Sea and the Black Sea and stretches into continental Europe. It is a mountainous country averaging about 1.000 meters in altitude. The topographic and climatic diversity of the region are important preconditions for the development of a rich and diverse fauna. Turkey is biogeographically one of the most interesting countries in the West Palaearctic region. The fauna of Hymenoptera of Turkey contains a large number of species in comparison with other Mediterranean countries, which are well known for their high biodiversity. The great richness and diversity of these families in the Turkish fauna is the result of the various topographic and climatic structure of the country. On the other hand, Turkey is a bound- 
ary of East Mediterranean, Sumerian and Irano-Turanian provinces of Palearctic Region that caused the richness of the fauna (YILDIRIM 2016).

The area of Bingöl province is $8,125 \mathrm{~km}^{2}$ and Diyarbakır province is $15,355 \mathrm{~km}^{2}$, both of them are mountaneous areas of South Eastern region of the country (Figs 1 and 2). The highest peak is the Bingöl Mountain with approximately 3,200 meters. The largest rivers are River Murat in Bingöl and River Tigris Diyarbakır province. The investigated area belong partly to the Eastern Anatolian Region(Bingöl) and the South-Eastern Anatolian Region (Diyarbakir). The climate is semi-arid continental, average monthty temperatures fluctuate between $-11^{\circ} \mathrm{C}$ and $+33^{\circ} \mathrm{C}$ which sometimes may reach the $-33^{\circ} \mathrm{C}$ and $+44^{\circ} \mathrm{C}$.

This paper aims to present new collection and biological data on Megalodontesidae, Argidae, Cimbicidae, Cephidae and Tenthredinidae (Symphyta: Hymenoptera) in Turkey.

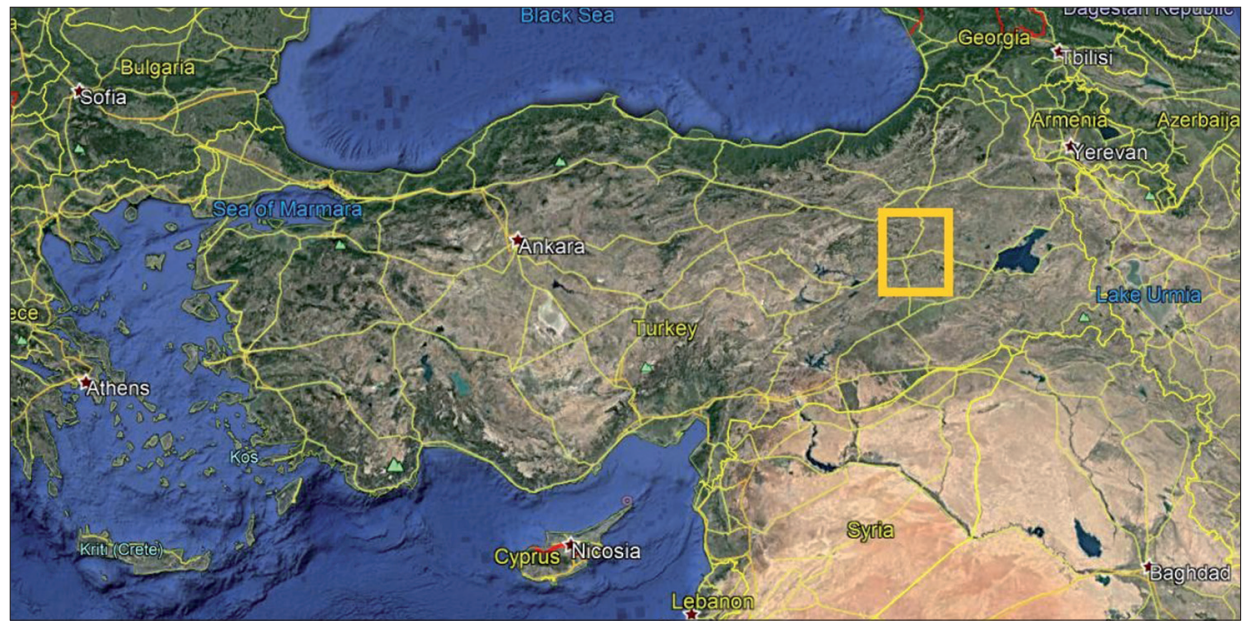

Fig. 1: Position of the investigated area in Turkey

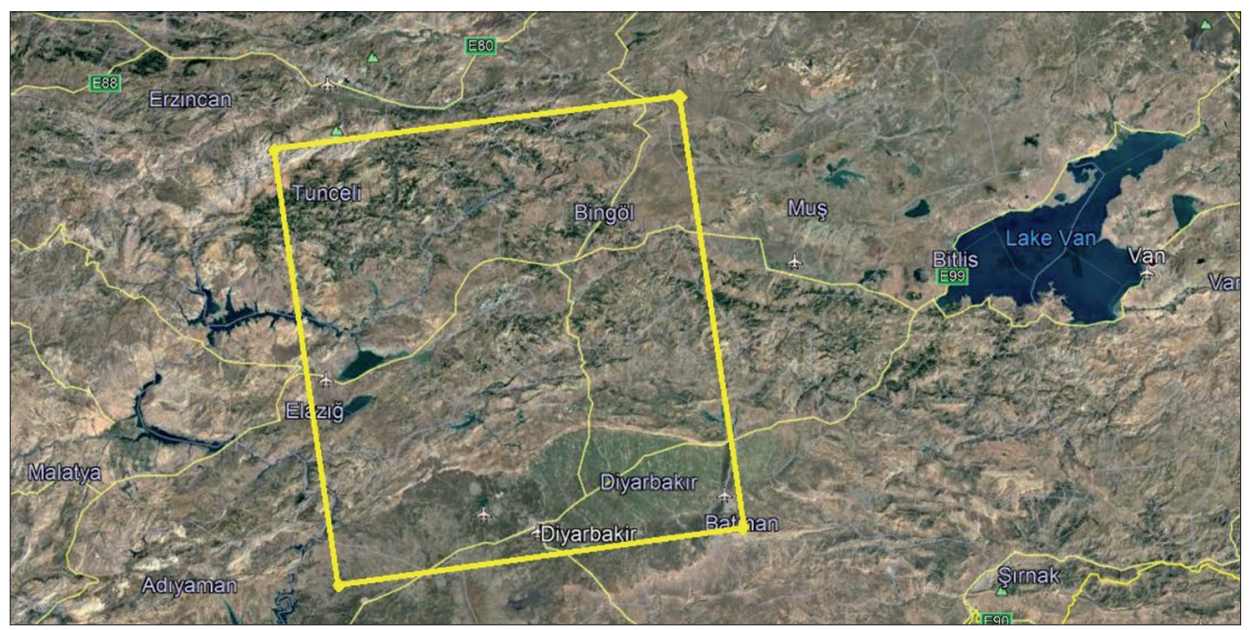

Fig. 2: Map of the investigated area 


\section{Material and methods}

The material of the Megalodontesidae, Argidae, Cimbicidae, Cephidae and Tenthredinidae (Symphyta: Hymenoptera) were collected from Bingöl and Diyarbakır provinces of Turkey between 2016 and 2018 (Figs. 3-6).

Material was collected by a variety of methods, mostly by sweeping grasses on meadows and pastures lands containing a variety of flowering plants. Provinces of the collected specimens are given in alphabetical order in the following list. The material is deposited partly in the Rippl-Rónai Museum, Kaposvár and partly in the Entomology Museum, Erzurum, Turkey (EMET) and in the Bingöl University, Faculty of Agriculture, Department of Plant Protection.

For the identification of the specimens we consulted the comprehensive works of ZHELOCHOVTSEV 1988 and BENSON 1968 completed with other papers as ENSLIN 1910a, b; TAEger 1992 and GussaKovskiJ 1935, 1947.

For the distribution and occurence of species in Turkey BENSON 1968, ÇALMAŞUR \& ÖzbeK 2004a,b, 2006; Çalmaşur 2006; Chevin \& Chenon 1982, Roller \& Haris, 2008 and ROLLER 1999 were consulted.

\section{Results}

In this study, two species from one genera of Megalodontesidae, eight species from two genera of Argidae, two species from one genera of Cimbicidae, three species from three genera of Cephidae and 23 species from six genera of Tenthredinidae are recorded from Turkey.

\section{Megalodontesidae}

Megalodontes cephalotes (Fabricius, 1781)

Material examined: Diyarbakır: Çermik, N $38^{\circ} 07^{\prime}$ 52.06", E 39 28' 38.78", 767 m, 13.IV.2018, 1 female,

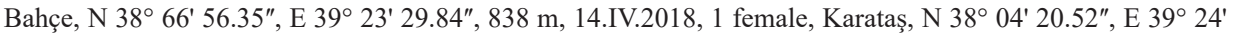
17.08", 677 m, 13.IV.2018, 1 female, Kartaltaşı, N 38 03' 50.68", E 39 23' 43.86", 712 m, 13. IV.2018, 1 female, Karakaya, N $38^{\circ} 03^{\prime}$ 24.42", E 39 18' 54.30", 618 m, 13.IV.2018, 1 female, Sinek, N 38 08' 46.01", E $39^{\circ} 26^{\prime} 26.84^{\prime \prime}, 827$ m, 14.IV.2018, 1 female, N $38^{\circ}$ 68' 57.08", E 39 27' 03.05", 835 m, 14.IV.2018, 1 female, Hani, Gürbüz, N $38^{\circ} 23^{\prime} 11.32^{\prime \prime}$, E $40^{\circ} 22^{\prime} 24.49^{\prime \prime}, 1037$ m, 11.V.2017, 1 female. Frequent. Known hostplant: Peucedanum cervaria.

\section{Megalodontes phaenicius (Lepeletier, 1823)}

Material examined: Bingöl: Karlıova, Kazanl1, N 38 23' 10.37", E 40 54' 34.28", 1794 m, 06.VI.2018 m, 1 male; Yayladere, Yaylabağ, N 39॰ 11' 11.13", E 40 06' 17.29", 1379 m, 13.VI.2018, 1 male. Frequent. Hostplant unknown.

\section{Argidae}

Arge beckeri Tournier, 1889

Material examined: Diyarbakır: Bismil, Başaklı, N $37^{\circ}$ 49' 48.02", E 40² 27' 51.59", 562 m, 30.III.2018, 1 male; Çüngüş, Güneydere, N 38 13' 43.21", E 39 17' 39.27", 1119 m, 19.IV.2018, 1 male; Hazro, Bağyurdu, N $38^{\circ} 14^{\prime} 13.92^{\prime \prime}$, E 40 49' 59.88", 995 m, 27.IV.2018, 1 male; Lice, Çavundur, N 38 19' 47.72", E 40³9' 59.81", 1183 m, 25.IV.2018, 1 male, Dolunay, N 38 19' 18.60", E 40 40' 30.57", 1007 m, 25.IV.2018, 1 male,

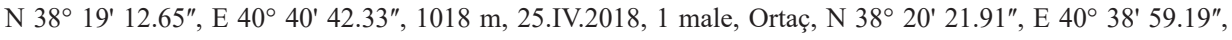
1040 m, 25.IV.2018, 1 male. Frequent. Hostplant: Euphorbia spp. 


\section{Arge cyanocrocea (Förster, 1771)}

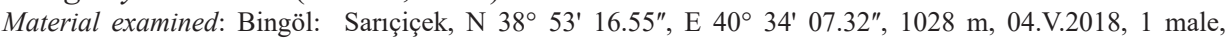
Yeşilköy, N 38 50' 11.55", E 40² 29' 52.18", 1254 m, 06.V.2018, 1 male. Frequent. Known hostplant: Rubus idaeus.

\section{Arge frivaldszkyi (Tischbein, 1852)}

Material examined: Diyarbakır: Kocaköy, Ambar, N $38^{\circ} 16^{\prime}$ 07.99", E 40²8' 29.61", 733 m, 24.IV.2018, 1 female; Lice, Beni, N $38^{\circ} 20^{\prime}$ 07.29", E 40³8' 57.00", 1124 m, 25.IV.2018, 1 female, Kutlu, N $38^{\circ} 21^{\prime} 37.62^{\prime \prime}$, E 40 46' 28.39", 825 m, 28.IV.2018, 1 female, Oyuklu, N 38 19' 44.71", E 40 45' 31.90", 939 m, 28.IV.2018, 1 female,Yalaza, N $38^{\circ} 20^{\prime}$ 16.33", E 40 40' 35.45", 921 m, 25.IV.2018, 1 female. Frequent. Hostplant unknown.

\section{Arge melanochra (Gmelin, 1790)}

Material examined: Bingöl: Garip, N $38^{\circ} 47^{\prime}$ 10.02", E 40 32' 58.95", 993 m, 20.V.2018, 1 female, Köklü, N $38^{\circ} 55^{\prime} 38.44^{\prime \prime}$, E $40^{\circ} 38^{\prime} 40.46^{\prime \prime}, 1088$ m, 19.V.2018, 1 female; Genç, Çayağz1, N $38^{\circ} 47^{\prime} 16.15^{\prime \prime}$, E 40 53' 11.72", 1037 m, 19.V.2018, 1 female, Doğanevler, N $38^{\circ} 46^{\prime}$ 14.84", E 40 51' 33.19", 1419 m, 10.V.2018, 1

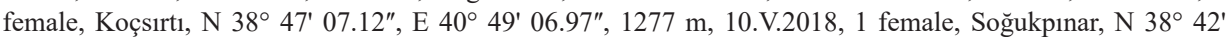
59.41", E 40²7' 11.55", 1032 m, 15.V.2018, 1 female, Yenisu, N 38 45' 17.43", E 40 52' 44.61", 1605 m, 10.V.2018, 1 female; Diyarbakır: Kardeşler, N $38^{\circ}$ 55' 34.84", E 40³8' 20.73", 1082 m, 17.V.2018, 1 female, Lice, Savat Bucağ 1 , N $38^{\circ} 20^{\prime}$ 44.50", E 40 37' 46.50", 1110 m, 25.IV.2018, 1 female. Common species. Hostplant: Crataegus oxycantha.

\section{Arge ochropus (Gmelin, 1790)}

Material examined: Diyarbakır: Lice, Tepe, N $38^{\circ} 23^{\prime} 34.56^{\prime \prime}$, E 40 43' 18.26", 835 m, 28.IV.2018, 1 male; Genç, Doğanay, N $38^{\circ} 42^{\prime} 26.73^{\prime \prime}$, E 40 32' 33.88", 1179 m, 14.V.2018, 1 male. Pest of Rosa spp. Locally frequent.

\section{Arge rustica (Linné, 1758)}

Material examined: Bingöl: Genç, Çanakal, N 38 45' 55.39", E 40 43' 58.57", 1530 m, 26.V.2017, 1 male. Sporadic. Hostplant: Quercus spp.

\section{Arge scita (Mocsáry, 1880)}

Material examined: Diyarbakır: Çermik, Bahçe, N $38^{\circ} 07^{\prime}$ 14.17", E 40² 23' 50.60", 898 m, 27.IV.2017, 1 female; Lice, Dallica, N $38^{\circ} 24^{\prime}$ 02.59", E 40 48' 43.40", 826 m, 28. 04. 2018, 1 male, Tepe, N 38 $22^{\prime} 23.07^{\prime \prime}$, E $40^{\circ} 44^{\prime} 32.11^{\prime \prime}, 801 \mathrm{~m}, 28 . I V .2018,1$ male; Silvan, Eskiocak, N $38^{\circ} 07^{\prime} 11.23^{\prime \prime}$, E $41^{\circ} 06^{\prime} 41.56^{\prime \prime}, 850$ m, 29.IV.2018, 1 female. Frequent. Prunus amygdalus.

Sterictiphora furcata (Villers, 1789)

Material examined: Bingöl: Solhan, Dilektepe, N $38^{\circ} 37^{\prime}$ 04.70", E 40 59' 47.74", 1288 m, 24.V.2018, 1 male. Frequent. Known hosptlant: Rubus idaeus.

\section{Cimbicidae}

Corynis orientalis (Konow, 1898)

Material examined: Diyarbakır: Çüngüş, Eskiocak, N $38^{\circ}$ 13' 55.18", E 39 15' 05.18", 864 m, 20.IV.2018, 1 female. Sporadic. Hostplant unknown.

Corynis crassicornis (Rossi, 1790)

Material examined: Bingöl: Kurudere, N 38 54' 39.88", E 40²8' 31.69", 1143 m, 07.V.2016, 1 female. Sporadic. Hosts are Sedum spp. (S. album, S. acre and S. sexangulare).

\section{Cephidae}

Calameuta (Calameuta) idolon (Rossi, 1794)

Material examined: Diyarbakır: Lice, Bağlan, N $38^{\circ} 20^{\prime} 03.91^{\prime \prime}$, E 40 43' 41.69", 835 m, 28.IV.2018, 1 female. Frequent. Hostplant unknown. 


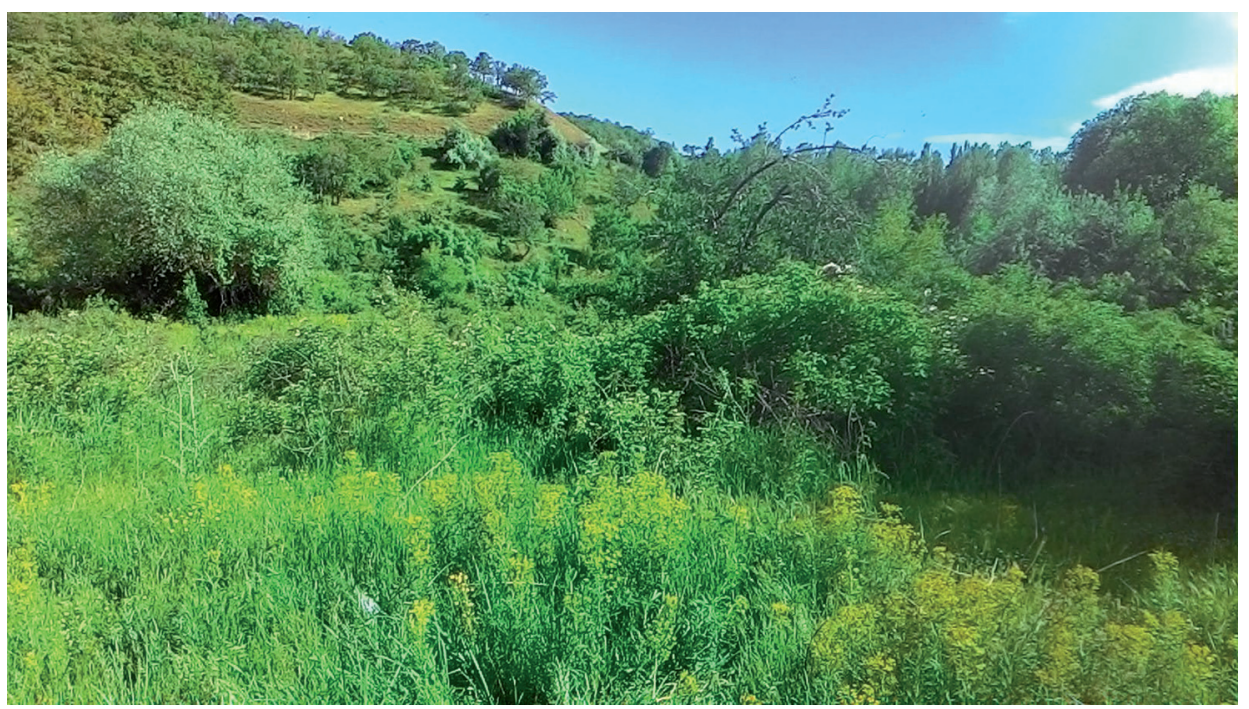

Fig. 3: Landscape in Solhan county

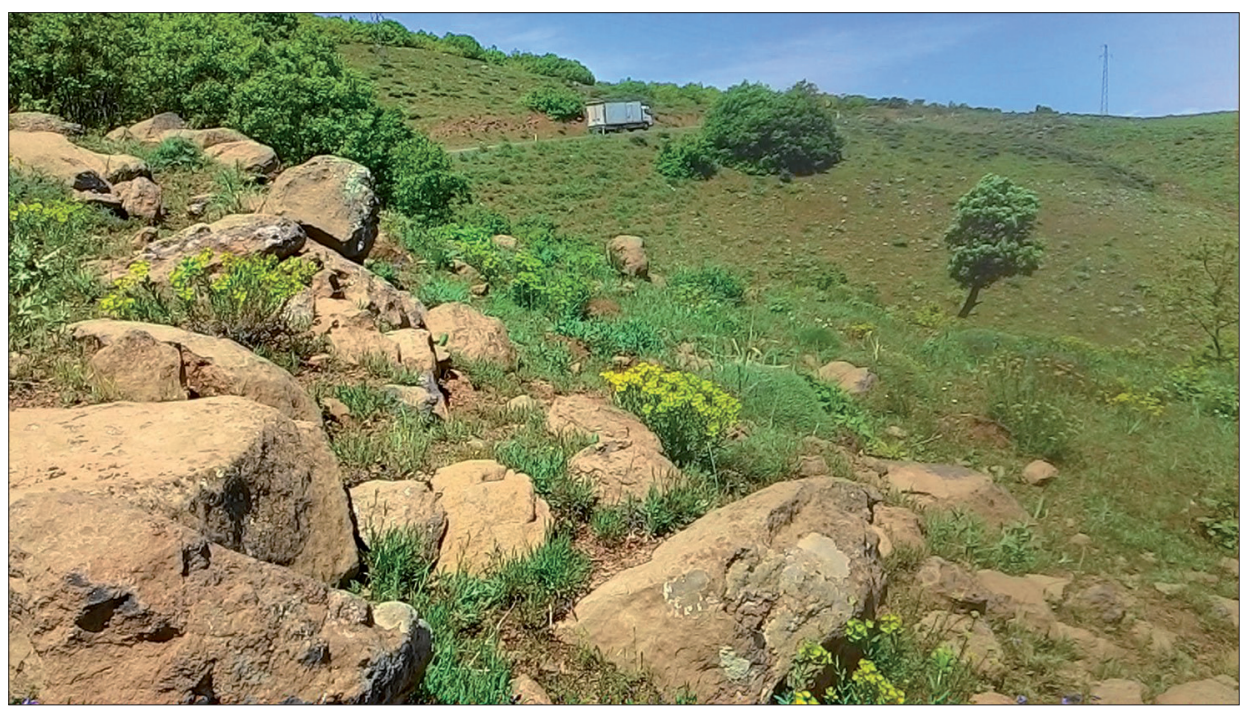

Fig. 4: Collecting site in Sancak

Syrista parreyssii (Spinola, 1843)

Material examined: Bingöl: Adakl1, Donattepe, N 39²12' 18.88", E 40²8' 01.49", 1321 m, 29.V.2017, 1 female. Frequent. Larva on Rosa spp.

Cephus pygmeus (Linné, 1767)

Material examined: Bingöl: Karlova, N 39 17' 28.27", E 40 59' 22.94", 1883 m, 12.VII.2016, 1 female. Generally common. Pest of cereals and grasses. 


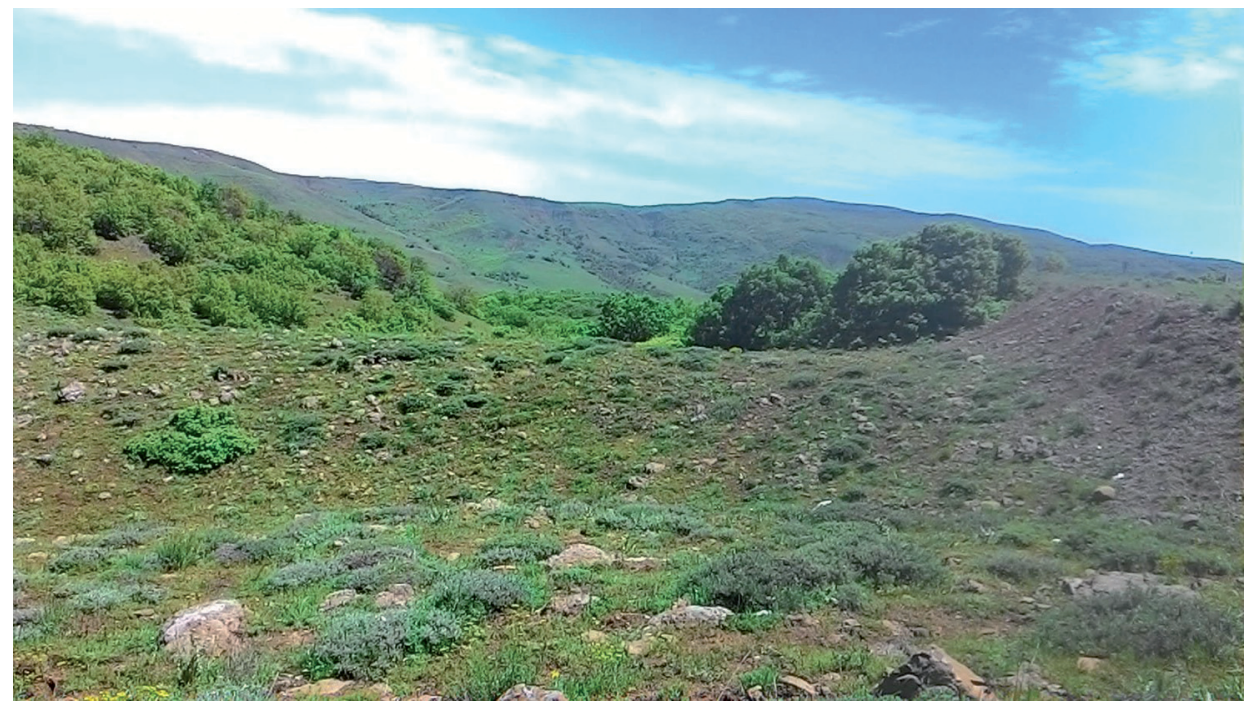

Fig. 5: Collecting site in Adaklı

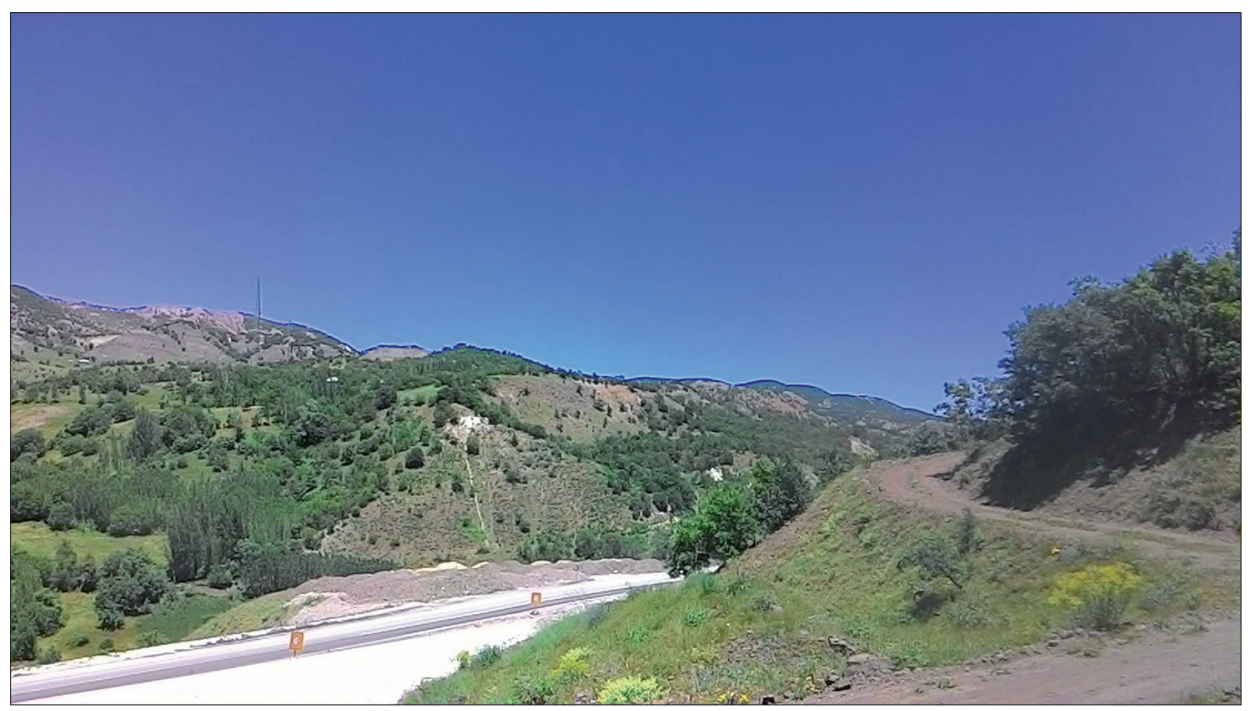

Fig. 6: Lice county in Diyarbakır province

\section{Tenthredinidae}

\section{Dolerinae}

Dolerus (Dicrodolerus) vestigialis (Klug, 1818)

Material examined: Bingöl: Yedisu, Elmalı, N 39² 22' 04.62", E 40³9' 12.28", 1885 m, 06.VI.2018, 1 female. Sporadic. Hostplants: Equisetum palustre, E. sylvaticum, E. arvense and E. pratense.

\section{Allantinae}

Athalia rufoscutellata Mocsáry, 1879

Material examined: Bingöl: Çeltiksuyu, N 38 51' 15.29", E 40 33' 30.58", 1015 m, 04.V.2018, 1 male; 
Karlıova, Yenidal, N $39^{\circ}$ 15' 23.88", E 41 14' 39.77", 1589 m, 04.VI.2018, 1 female. Frequent. Hostplant unknown.

\section{Blennocampinae}

Eutomostethus gagathinus (Klug, 1816)

Material examined: Diyarbakır: Çermik: Sinek, N $38^{\circ} 09^{\prime}$ 26.61", E 39 26' 40.60", 858 m, 27.IV.2017, 1 female. Sporadic. Hostplant unknown.

\section{Tenthredininae}

Macrophya (Macrophya) annulata (Geoffroy, 1785)

Material examined: Bingöl: Köklü, N 38 55' 52.81", E 40 38' 31.61", 1086 m, 17.V.2018, 1 female; Sancak, Nacakl1, N $38^{\circ}$ 10' 05.38", E 40² 21' 33.32", 1537 m, 26.V.2018, 1 female. Frequent. Larva on Potentilla reptans, Origanum vulgare, Euphorbia, Rosa, Rubus and Sambucus spp.

\section{Macrophya (Macrophya) diversipes (Schrank, 1782)}

Material examined: Bingöl: Çayboyu, N $38^{\circ}$ 53' 57.77", E 40 30' 52.08", 1083 m, 05.V.2018, 1 female, Çeltiksuyu, N $38^{\circ}$ 51' 15.29", E 40 33' 30.58", 1015 m, 04.V.2018, 1 female, Çukurca, N 38 10' 35.30", E $40^{\circ} 31^{\prime}$ 17.62", 1215 m, 05.V.2018,1 female; Adaklı, Erbaşlar, N 39॰ 10' 44.79", E 40 31' 58.78", 1525 m, 29.V.2016, 1 female; Genç, Yalya Bucag1, N $38^{\circ} 38^{\prime}$ 26.33", E 40³1' 58.59", 1388 m, 14.V.2018, 1 male; Karlıova, Dörtyol, N $39^{\circ} 22^{\prime} 33.58^{\prime \prime}$, E 40³0' 49.96", 1813 m, 06.VI.2018, 1 female, Suçatı, N 39²3' 07.16", E 40 54' 53.52", 1800 m, 06.VI.2018, 1 female; Kiğı: Yeşilyurt, N 39 16' 58.79", E 40² 21' 24.37", 1322 m, 12.VI.2018, 1 male; Solhan: Merkez, N $38^{\circ}$ 57' 06.75", E 41 02' 04.00", 1321 m, 24.V.2018, 1 female,

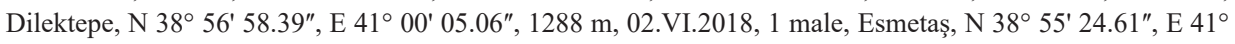
07' 52.53", 1703 m, 31.V.2018, 1 female, Sudüğünü, N 39 03' 10.23", E 40 25' 38.99", 1618 m, 26.V.2018, 1 male, Yiğitharmanı, N $38^{\circ} 57^{\prime} 59.87^{\prime \prime}$, E $40^{\circ} 56^{\prime} 50.61^{\prime \prime}, 1380$ m, 24.V.2018, 1 female; Yedisu, Kabaoluk, N $39^{\circ} 25^{\prime}$ 52.06", E 40²9' 48.17", 1409 m, 06.VI. 2018 m, 1 female. Frequent. Hostplant unknown.

\section{Macrophya mocsaryi W.F. Kirby, 1882}

Material examined: Bingöl: Yayladere, Saritosun, N $39^{\circ} 12^{\prime} 24.89^{\prime \prime}$, E 40 05' 10.12", 1421 m, 13.V.2018,1 female. Rare. Hostplant unknown.

\section{Macrophya (Macrophya) oedipus Benson, 1968}

Material examined: Bingöl: Kardeşler, N 38 54' 53.33", E 40 38' 20.73", 1082 m, 17.V.2018, 1 female. Sporadic. Hostplant unknown. Known only from Turkey: Amasya.

\section{Macrophya (Macrophya) blanda (Fabricius, 1775)}

Material examined: Bingöl: Kardeşler, N $38^{\circ}$ 54' 53.33", E 40 37' 24.74", 1068m, 17.V.2018, 1 female; Karlıova, Kaynarpınar, N $39^{\circ} 22^{\prime}$ 29.88", E 40 44' 21.98", 1885 m, 28.V.2017, 1 female; Yedisu, Gelinpertek, N $39^{\circ} 27^{\prime}$ 05.31", E 40²3' 55.83", 1548 m, 28.V.2018, 1 female. Frequent. Hostplant unknown.

\section{Macrophya postica Brullé, 1832}

Material examined: Bingöl: Büyükterkören, N $38^{\circ}$ 50' 35.45", E 40 34' 13.30", 1017 m, 20.V.2018, 1 female, Çayağz1, N $38^{\circ} 47^{\prime} 44.47^{\prime \prime}$, E 40 33' 15.35", 998 m, 20.V.2018, 1 female, N 38 48' 12.13", E 40³3' 21.30",

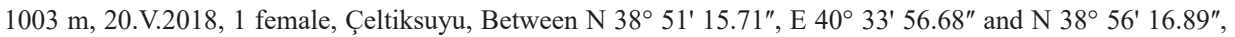
E 40 33' 45.63", 1015 m, 04.V.2018, 2 females, 4 males, Çiçekyayla, N 38 49' 21.94", E 40 28' 11.63", 1442 m, 27.V.2017, 1 female, Dicle, N $37^{\circ}$ 52' 06.13", E 40 35' 45.41", 608 m, 30.III.2018, 1 female, Düzyayla, N

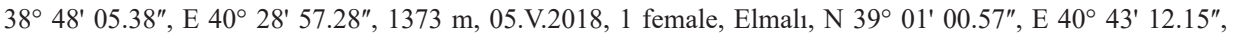

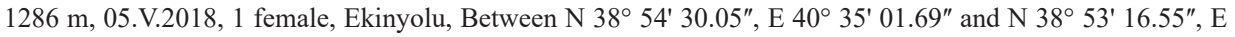
$40^{\circ} 34^{\prime}$ 07.32", 1028-1047 m, 19.V.2018, 1 female;.Çüngüş: Kayapınar, N 38 18' 09.50", E 39 12' 34.57", 904 m, 20.IV.2018, 1 female, N $38^{\circ}$ 54' 13.80", E 40 35' 10.08", 1040m, 17.V.2018, 1 female, 1 male, Gümüşlü, N $38^{\circ} 45^{\prime}$ 66.81", E 40²8' 49.70", 1087 m, 06.V.2018, 1 female, Garip, N 38 46' 50.09", E 40³3' 17.79", 992 m, 20.V.2018, 1 female, Güzelköy, N 3800' 59.45", E 40 15' 14.69", 594 m, 29.III.2018, 1 female, Kaleönü, N $38^{\circ}$ 54' 08.82", E 40 32' 33.71", 1040 m, 05.V.2018, 1 female, N $38^{\circ} 53^{\prime}$ 53.74", E 40 33' 25.10", 1031 0. 05.V.2018, 1 female, Kardeşler, N $38^{\circ}$ 54' 53.33", E 40 37' 24.74" 1068 m, 17.V.2018, 1 
female, N $38^{\circ}$ 54' 53.33", E 40 37' 27.47" and N $38^{\circ} 55^{\prime} 34.84^{\prime \prime}$, E 40 38' 20.73", 1068-1082 m, 19.V.2018, 1 female, Kartal, N $39^{\circ} 01^{\prime} 35.75^{\prime \prime}$, E 40² 23' 32.24", 1676 m, 26.V.2018, 1 female, Kılçadır, N $38^{\circ} 46^{\prime} 21.51^{\prime \prime}$, E $40^{\circ} 28^{\prime}$ 51.09", 1160 m, 06.V.2018, 1 male, Küçükterkören, N $38^{\circ}$ 51' 18.33", E 40²9' 58.29", 1186 m, 06.V.2018, 1 female; Genç, N $38^{\circ}$ 44' 07.30", E 40³0' 35.06", 986 m, 15.V.2018, 1 female, Ardıçdibi, N $38^{\circ}$ 46' 32.40", E 40 36' 40.02", 1054 m, 09.V.2018, 1 female, Binekli, N $38^{\circ} 45^{\prime} 47.45^{\prime \prime}$, E $40^{\circ} 53^{\prime} 23.88^{\prime \prime}, 1436$ m, 10.V.2018, 1 female, Çayağzı, N 38 46' 30.25", E 40 33' 16.05", 980 m, 09.V.2018, 1 male, Dikköy, N

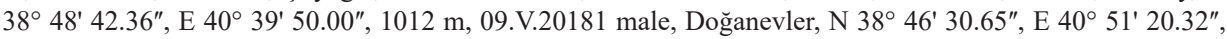
1419 m, 10.V.2018, 1 male, Meşedalı, N $38^{\circ} 47^{\prime}$ 07.96", E 40 37' 37.11", 1001 m, 09.V.2018, 1 female,

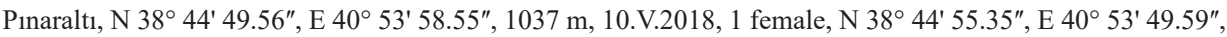
1569 m, 10.V.2018, 1 female, Şehitköy, N 38 42' 04.23", E 40³0' 41.61", 1147 m, 15.IV.2018, 1 female, Sürekli, N $38^{\circ}$ 46' 05.76", E 40³5' 39.06", 1010 m, 09.V.2018, 2 females, Yağızca, Between N 38 49' 18.61", E 40 46' 47.03", and N $38^{\circ} 48^{\prime} 31.78^{\prime \prime}$, E 40 45' 32.52", 1121-1330 m, 10.V.2018, 1 male, N $38^{\circ} 48^{\prime} 41.26^{\prime \prime}$, E $40^{\circ} 45^{\prime}$ 15.59", 1152 m, 10.V.2018, 1 female, Yayla Bucağı, N $38^{\circ} 38^{\prime} 18.36^{\prime \prime}$, E 40 31' 39.92", 1342 m, 14.V.2018, 1 male; Karlıva, Buncukgöze, N 39 14' 12.70", E 40 58' 39.08", 1760 m, 03.VI.2018, 1 male,

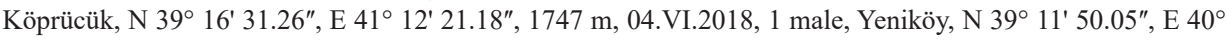
56' 07.35", 1748 m, 03.VI.2018, 1 female; Solhan, N $38^{\circ}$ 56' 23.25", E 41 08' 18.56", 1727 m, 02.VI.2018, 1

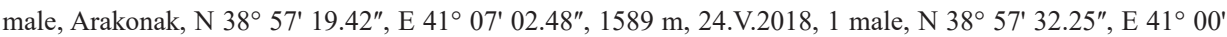
51.26", 1338 m, 24. 05. 2018, 1 female, Arakonak, N 38 57' 38.93", E 41º 07' 02.91", 1589 m, 24.V.2018, 1

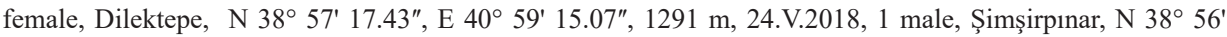

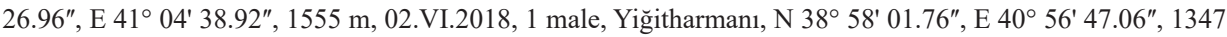
m, 24.V.2018, 1 male; Diyarbakır: Bismil, Köseli, N $37^{\circ}$ 50' 51.99", E 40 38' 49.64",558. 30.III.2018, 1 female; Çermik, Aşağışeyhler, N $38^{\circ}$ 10' 28.47", E 410 07' 02.48", 693 m, 14.IV.2018, 1 male, Karakoluk, N

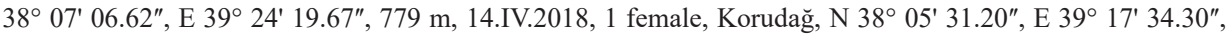
897 m, 13.IV.2018, 1 male, Seyhandede, N 38 02' 03.86", E 39 17' 23.50", 615 m, 15.IV.2018, 1 female, Sinek, N $38^{\circ}$ 68' 57.08", E 39² 27' 03.05", 695 m, 14.IV.2018, 1 female; Çınar, Dikmencik, N $37^{\circ} 45^{\prime} 23.50^{\prime \prime}$, E 40²1' 00.81", 669 m, 30.III.2018, 1 female; Eğil, Selmanköy, N $38^{\circ} 11^{\prime} 45.04^{\prime \prime}$, E 40 08' 46.13", 809 m, 12. 05. 2017, 1 female; Ergani, Ahmetli Bucağı, N $38^{\circ} 10^{\prime}$ 56.21", E 39॰ 52' 13.44", 793 m, 22.IV.2018, 1 female, Sağırlı, N $38^{\circ} 21^{\prime}$ 58.75", E 39 48' 23.44", 1018 m, 22.IV.2018, 1 female, Yolköprü, N 38 15' 03.79", E $39^{\circ} 42^{\prime}$ 03.03", 864 m, 21.IV.2018, 1 female. Common. Hostplant unknown.

\section{Macrophya (Macrophya) superba Tischbein, 1852}

Material examined: Bingöl: Karlıva, Kıraçtepe, N 39 12' 33.66", E 40 57' 24.32", 1734 m, 03.VI.2018, 1 male, Ulupınar, N $39^{\circ} 22^{\prime}$ 44.70", E 40 56' 47.05", 1808 m, 06.VI.2018, 1 female; Yedisu, Şenköy, N 39²5' 13.69", E 40 31' 52.37", 1496 m, 06.VI.2018, 1 male. Frequent. Hostplant unknown.

\section{Tenthredo (Cephaledo) bifasciata ssp. bifasciata O. F. Müller, 1766}

Material examined: Bingöl: Çukurca, N 38 55' 13.31", E 40 31' 17.62", 1315 m, 05.V.2018, 1 female, Ekinyolu, N $38^{\circ}$ 53' 53.54", E 40 34' 31.81", 1033 m, 17.V.2018, 1 male, Elmali, N 390 01' 00.57", E 40 43' 12.15", 1286 m, 05.V.2018, 1 male, Kaleonü, N $38^{\circ}$ 53' 53.74", E 40 33' 25.10", 1031 m, 05.V.2018, 1 female, Kardeşler, N $38^{\circ}$ 55' 34.84", E 40 38' 20.73", 1082 m, 17.V.2018, 1 male, N 38 56' 05.09", E 40 39' 00.49", 1107 m, 17.V.2018, 1 female, Yenibaşlar, N 38 59' 50.35", E 40 41' 37.75", 1177m, 17.V.2018, 1 female, N

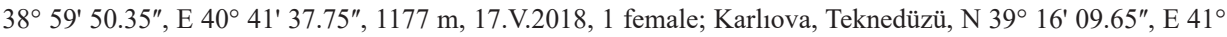
13' 20.05", 1691 m, 04.VI.2018, 1 male, Sancak, N 39 03' 55.46", E 40²2' 46.53", 1569 m, 26.V.2018, 1

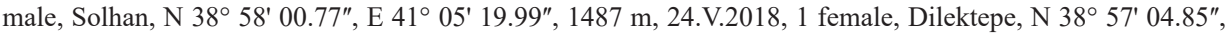

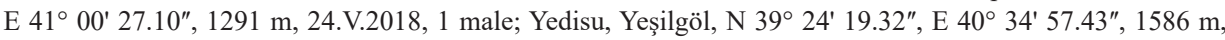
06.VI.2018, 1 male; Diyarbakır: Çermik, Karataş, N 38 04' 20.52", E 39 24' 17.08", 677m, 13.IV.2018, 1 female; Dicle: Bozbaba, N $38^{\circ} 20^{\prime} 14.51^{\prime \prime}$, E 40 06' 21.15", 829 m, 20.IV.2018, 1 female, Biçer, N $38^{\circ} 21^{\prime}$ 59.34", E 40 01' 33.98", 957 m, 20.IV.2018, 1 female; Eğil, Ulubaş, N $38^{\circ} 18^{\prime}$ 57.92", E 40 02' 15.77",839 m, 19.IV.2018, 1 female; Hani, An1l, N $38^{\circ} 24^{\prime}$ 01.60", E 40²0' 37.48", 896 m, 21.IV.2018, 1 female, Bademli, N $38^{\circ}$ 19' 42.62", E 40 17' 17.01", 754 m, 21.IV.2018, 2 males, Başköy, N 38²2' 44.54", E 40 14' 05.89", 822 m, 21.IV.2018, 1 male, Belen, N 38²5' 09.41", E 40²2' 05.00", 962 m, 21.IV.2018, 1 male, Döğer, N $38^{\circ} 21^{\prime} 59.83^{\prime \prime}$, E $40^{\circ} 13^{\prime}$ 08.19", 750 m, 21.IV.2018, 1 female; Lice, Angül, N 38 23' 13.08", E 40 32' 56.04", 877 m, 15.IV.2018, 1 female. Frequent. Hostplant unknown, probably Aegopodium. 
Tenthredo (Cephaledo) costata Klug, 1817

Material examined: Bingöl: Çayboyu, N 38 53' 57.77", E 40 30' 52.08", 1083 m, 05.V.2018, 1 female, Çeltiksuyu, N $38^{\circ} 51^{\prime} 15.29^{\prime \prime}$, E 40 33' 30.58", 1015 m, 04.V.2018, 1 male, Ekinyolu, N $38^{\circ} 53^{\prime} 53.54^{\prime \prime}$, E $40^{\circ}$ 34' 31.81", 1033 m, 17.V.2018, 1 male, Kaleönü, N 38 53' 53.74", E 40 33' 25.10", 1031 m, 05.V.2018, 1

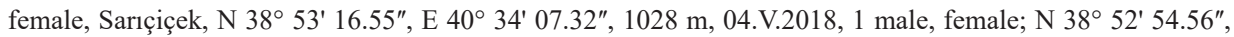
E 40 34' 02.26", 1025 m, 04.V.2018, 1 male, N 38 54' 06.79", E 40 36' 37.86", 1059 m, 17.V.2018, 1 female; Genç, Kumgeçit, N $38^{\circ}$ 47' 45.84", E 40 37' 57.35", 1003 m, 09.V.2018, 1 male, Savuran, N 38 43' 13.69",

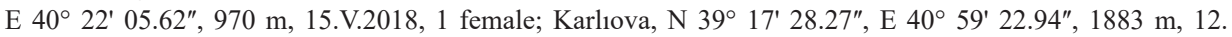

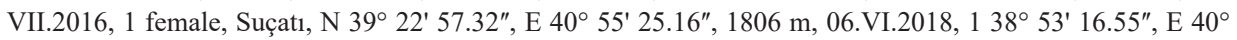

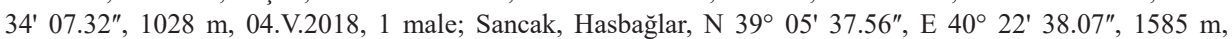
26.V.2018, 1 male, N $39^{\circ} 11^{\prime} 00.95^{\prime \prime}$, E $40^{\circ} 21^{\prime} 56.60^{\prime \prime}, 1558$ m, 26.V.2018, 1 female. Frequent. Hostplant unknown.

\section{Tenthredo (Cephaledo) excellens (Konow, 1886)}

Material examined: Bingöl: Kaleönü, N 38 54' 08.82", E 40 32' 33.71", 1040 m, 05.V.2018, 2 females,

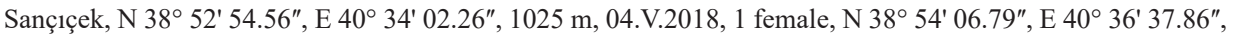
1059 m, 17.V.2018, 1 female, N 38 53' 30.30", E 40 35' 48.36", 1042 m, 19.V.2018, 1 female; Adakl1, Karaçubuk, N $39^{\circ} 11^{\prime} 52.69^{\prime \prime}$, E $40^{\circ} 28^{\prime} 20.28^{\prime \prime}, 1408$ m, 11.VI.2018, 1 female, Kozlu, N $39^{\circ} 18^{\prime} 18.40^{\prime \prime}$, E $41^{\circ}$ 09' 28.08", 1494 m, 29.V.2017,1 female; Karlıova, Alabalık, N 39 18' 18.40", E 41 09' 28.08", 1785 m, 04. VI.2018, 1 female, Kargapazarı, N 39 18' 47.67", E 41 06' 52.87", 1860 m, 04.VI.2018, 1 female; Yedisu,

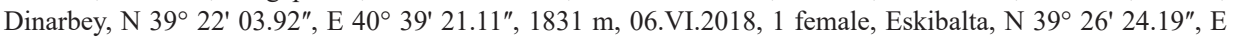
$40^{\circ} 28^{\prime} 16.59^{\prime \prime}, 1415$ m, 06.VI.2018, 1 female. Frequent. Hostplant unknown.

\section{Tenthredo (Elinopsis) vittata (Kriechbaumer, 1869)}

Material examined: Diyarbakır: Çermik, N $38^{\circ} 07^{\prime} 52.06^{\prime \prime}$, E 39 $29^{\circ} 38.78^{\prime \prime}, 767$ m, 13.IV.2018, 1 female,

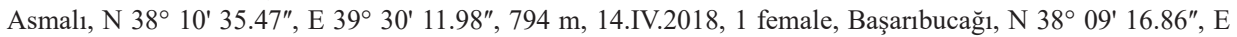
$39^{\circ} 34^{\prime} 28.52^{\prime \prime}, 699$ m, 15.IV.2018, 1 female, Kartaltaşı, N $38^{\circ}$ 03' 56.22", E 39 23' 16.96", 593 m, 13.IV.2018, 1 female, Pinarlı, N $38^{\circ} 06^{\prime} 10.31^{\prime \prime}$, E $39^{\circ} 17^{\prime} 59.07^{\prime \prime}, 1077$ m, 14.IV.2018, 1 female; Kulp, Çukurca, N $38^{\circ}$ 23' 39.40", E 40 56' 54.22", 982 m, 29.IV.2018, 1 female; Silvan, Gündüz, N 38 16' 29.97", E 40 65' 10.39", 911 m, 29.IV.2018, 1 male, Samanyurdu, N 38 05' 26.46", E 40 12' 51.69", 611 m, 29.IV.2018, 1 female, Susuz, N $38^{\circ} 10^{\prime} 22.80^{\prime \prime}$, E 405 55' 50.61", 919 m, 29.IV.2018, 1 female. Frequent. Hostplant unknown.

\section{Tenthredo (Elinora) baetica Spinola, 1843}

Material examined: Diyarbakır: Hazro, Mutluca, N 38 15' 41.58", E 40 53' 14.98", 971 m, 27.IV.2018,1 female. This species is newly recorded from Turkey. So far, it was known only from Spain and Portugal. Hostplant unknown.

\section{Tenthredo (Elinora) dahlii Klug, 1817}

Material examined: Diyarbakır:: Çermik, Asmalı, N 38 11' 25.32", E 39³ 33' 07.34", 793 m, 14.IV.2108, 1

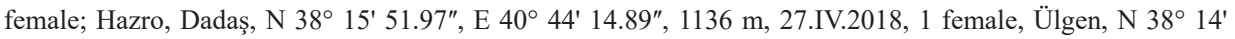
59.02", E 40 53' 17.26", 989 m, 27.IV.2018, 1 female; Kocaköy, Tepecik, N $38^{\circ} 16^{\prime} 05.49^{\prime \prime}$, E 40 33'03.61", 884 m, 25.IV.2018, 1 female, Yağızköy, N 38 18' 24.87", E 40 30' 20.70", 921 m, 24.IV.2018, 1 female; Kulp, Özbek, N $38^{\circ} 29^{\prime} 11.20^{\prime \prime}$, E $40^{\circ} 57^{\prime} 56.80^{\prime \prime}, 948$ m, 29.IV.2018, 1 female; Silvan, Çarıklı, N $38^{\circ} 03^{\prime} 31.08^{\prime \prime}$, E $41^{\circ} 11^{\prime} 32.52^{\prime \prime} 591$ m, 29.IV.2018, 1 female. Frequent. Hostplant unknown.

\section{Tenthredo (Paratenthredo) monozonus (Kriechbaumer, 1869)}

Material examined: Bingöl: Sarıçiçek, N 38 54' 06.49", E 40³6' 37.86", 1059 m, 17.V.2018, 1 female. Rare. Hostplant unknown.

\section{Tenthredo (Zonuledo) distinguenda (Stein, 1885)}

Material examined: Bingöl: Kırkağıl, N 38 54' 48.08", E 40² 22' 42.70", 1731 m, 27.V.2017, 1 male; Hazro, Bağyurdu, N 39॰ 14' 32.71", E 40 49' 08.85", 994 m, 27.IV.2018, 1 male; Kiğı: Nacakl1, N 39 10' 17.26", E $40^{\circ} 20^{\prime} 33.73^{\prime \prime}, 1439$ m, 29.V.2016, 1 female; Diyarbakır: Çermik, Yayıklı, N $38^{\circ} 03^{\prime} 52.46^{\prime \prime}$, E 39²0' 33.49", 
1119m, 19.IV.2018, 1 female; Ergani, Akçoban, N 37 55' 56.21", E 39 41' 19.67", 970 m, 27.IV.2017, 1 male. Frequent. Hostplant unknown.

Tenthredo (Zonuledo) flavipennis Brullé, 1832

Material examined: Bingöl: Adakl1, Donattepe, N 39 12' 17.51", E 40²7' 22.56", 1371 m, 11.VI.2018, 1 female. Sporadic. Hostplant unknown.

\section{Tenthredo (Zonuledo) nazareensis (André, 1881)}

Material examined: Diyarbakır: Yukarıkılıçtaşı, N $37^{\circ}$ 56' 49.73", E 40 14' 54.28", 597 m, 29.III.2018, 1 female; Çermik, Asmalı, N 38 11' 39.73", E 39³3' 07.34", 808 m, 14.IV.2018, 1 male, Karakaya, N 38 02' 48.67", E 39 19' 62.05", 553 m, 15.IV.2018, 1 male. Sporadic. Hostplant unknown.

\section{Tenthredo (Zonuledo) zonula Klug, 1817}

Material examined: Bingöl: Ağaçeli, N 38 55' 44.45", E 40 42' 18.73", 1257 m, 05.V.2018, 1 female, Çeltiksuyu, Between N $38^{\circ}$ 51' 15.71", E 40 33' 56.68" and N 38 56' 16.89", E 40 33' 45.63", 1013-1017m, 04.V.2018, 1 female, 3 males, Çukurca, N $38^{\circ}$ 55' 13.31", E 40 31' 17.62", 1250 m, 05.V.2018, 1 male, İncesu,

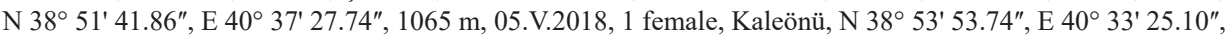

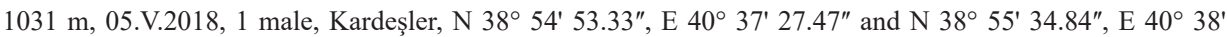
20.73", 1068-1082 m, 17.V.2018, 2 males, Köklü, N 38 55' 52.81", E 40 38' 31.61", 1086 m, 17.V.2018, 1 male, Sarıçiçek, Between N $38^{\circ} 53^{\prime} 16.55^{\prime \prime}$, E 40 34' 07.32" and N 38 52' 54.56", E 40 34' 02.06", 1025-1028 m, 04.V.2018, 1 male; Karlıova, N 39 16' 30.61", E 40 59' 54.17", 1806 m, 03.VI.2018, 1 female, Toklular, N $39^{\circ} 15^{\prime} 57.30^{\prime \prime}$, E $40^{\circ} 59^{\prime} 28.61^{\prime \prime}, 1787$ m, 03.VI.2018, 1 male; Kiğ 1 , Demirkanat, N $39^{\circ} 13^{\prime} 16.52^{\prime \prime}$, E $40^{\circ}$ $20^{\prime}$ 37.14", 1454 m, 12.VI.2018, 1 female; Solhan, Arakonak, N $38^{\circ}$ 57' 26.93", E 41 06' 54.36", 1612 m, 02.VI.2018, 1 female; Yayladere, Hasköy, N 39 12' 37.44", E 40 04' 28.96", 1406 m, 13.VI.2018, 2 males, Korlu, N $39^{\circ} 10^{\prime} 36.36^{\prime \prime}$, E 4009' 19.47", 1231 m, 13.VI.2018, 1 male, Yolgüdün, N $39^{\circ} 10^{\prime} 28.62^{\prime \prime}$, E 40 04' 20.21", 1509 m, 15.VII. 2017, 1 female; Diyarbakır: Güvercinlik, N $37^{\circ}$ 59' 06.08", E 40 14' 29.81", 613 m, 29.III.2018, 1 male Sivritepe, Between N $38^{\circ} 06^{\prime} 34.79^{\prime \prime}$, E $40^{\circ} 12^{\prime} 41.83^{\prime \prime}$ and N $38^{\circ} 06^{\prime} 30.86^{\prime \prime}$, E $40^{\circ} 13^{\prime}$ 19.38", 619-630 m, 29.III.2018, 1 female; Bismil, Ambar, N 37 50' 41.20", E 40 33' 30.69", 548 m, 30. III.2018, 1 female, Başaklı, N $37^{\circ}$ 49' 42.46", E 40² 28' 49.43", 558 m, 30.III.2018, 1 male, Göksü, N $37^{\circ} 50^{\prime}$ 07.51", E 40 32' 26.34", 598 m, 30.III.2018, 1 female, Sarılar, N 37 48' 53.53", E 40²3' 55.47", 593 m, 30.III.2018, 1 male; Çermik, Aşağışeyhler, N $38^{\circ}$ 10' 28.47", E 39 29' 21.77", 693 m, 14.IV.2018, 1 male, Asmalı, N $38^{\circ}$ 10' 35.47", E 39 30' 11.98", 791m, 14.IV.2018, 1 male, Başarıbucağ 1 , N 38 06' 00.76", E 39 34' 10.70", 743 m, 15.IV.2018, 1 female, Bahçe, N 38 06' 55.82", E 39 23' 43.79", 833 m, 14.IV.2018, 1 female, Gözerek, N $38^{\circ} 08^{\prime}$ 49.67", E 39॰ 33' 18.21",1052 m, 15.IV.2018, 1 female, Güzel, N $38^{\circ} 01^{\prime} 08.45^{\prime \prime}$, E $39^{\circ} 11^{\prime} 31.16^{\prime \prime}, 840$ m, 15.IV.2018, 1 female, Karakaya, N 38॰ 03' 24.42", E 39 18' 54.30", 618 m, 13. IV.2018, 1 male, Kartaltaşı, N $38^{\circ}$ 03' 50.68", E 39² 23' 40.86", 712 m, 13.IV.2018, 1 male, Kayağediği, N $38^{\circ}$ 09' 16.86", E 39 34' 28.52", 1085 m, 15.V.2018, 2 females, Kurudağ, N 38 05' 31.20", E 39 17' 34.30", 897 m, 13.IV.2018, 1 male, Toplu, N 38 06' 16.37", E 39 33' 48.02", 779 m, 15.IV.2018, 1 male; Çınar, Bozçalı, N $37^{\circ} 45^{\prime} 19.49^{\prime \prime}$, E 40 21' 08.98", 669 m, 30.III.2018, 1 female; Çüngüş, Between N 38 13' 11.54", E $39^{\circ}$ $17^{\prime} 38.99^{\prime \prime}$ and N $38^{\circ} 13^{\prime} 00.66^{\prime \prime}$, E 39 $17^{\prime} 33.85^{\prime \prime}, 980-1001 \mathrm{~m}, 19 . I V .2018,1$ male, Güneydere, N $38^{\circ} 13^{\prime}$ 43.21", E $39^{\circ}$ 17' 39.27", 1119 m, 19.IV.2018, 1 male, Kayarpınar, N $38^{\circ} 18^{\prime}$ 28.44", E 39॰ 11' 32.32", 756 m, 20.IV.2018, 1 male, Keleşevleri, N 38 11' 40.95", E 39² 21' 15.33", 968 m, 19.IV.2018, 1 male, Malkaya,

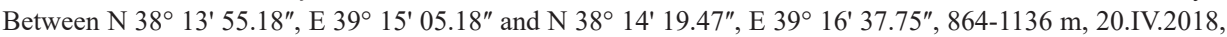
1 female, Oyuklu, N $38^{\circ} 12^{\prime}$ 27.82", E 39² 21' 58.48", 1000 m, 19.IV.2018, 1 female, Sağtepe, N 38 13' 41.26", E $39^{\circ} 22^{\prime} 33.16^{\prime \prime}, 1128 \mathrm{~m}, 19 . I V .2018,1$ male, Yenice, Between N $38^{\circ} 17^{\prime} 05.87^{\prime \prime}$, E $39^{\circ} 15^{\prime} 28.49^{\prime \prime}$ and N $38^{\circ}$ 16' 44.83", E 39॰ 15' 09.29", 1209-1304 m, 20.IV.2018, 1 female, 1 male; Eğil, Ulubaş, N $38^{\circ} 18^{\prime}$ 57.60", E 40 02' 14.45", 792 m, 19.IV.2018, 1 female; Hazro: Dadaş, N 38 16' 00.95", E 40 44' 32.71", 1169 m, 27.IV.2018, 1 male. Common. Hostplant: Hypericum perforatum.

\section{Tenthredopsis annuligera (Eversmann, 1847)}

Material examined: Diyarbakır: Çermik, Karamusa, N 38 09' 32.42", E 39³0' 28.84", 909 m, 27.IV.2017, 1 female. Sporadic. Hostplant unknown. 
Tenthredopsis albonotata (Brullé, 1832)

Material examined: Diyarbakır: Cermik, Gözerek, N 38 09' 18.84", E 39 33' 36.50", 948 m, 15.IV.2018, 1 male; Dicle, Kocaalan, N $38^{\circ} 21^{\prime} 39.51^{\prime \prime}$, E 40 07' 09.51", 806 m, 20.IV.2018, 1 female, Hazro, Ormankaya, N $38^{\circ} 17^{\prime} 10.83^{\prime \prime}$, E $40^{\circ} 47^{\prime} 46.39^{\prime \prime}, 1077$ m, 27.IV.2018, 1 female; Lice, Yalaza, N $38^{\circ} 20^{\prime} 00.71^{\prime \prime}$, E 40 $40^{\prime}$ 56.36", 929 m, 25.IV.2018, 1 male. Frequent. Hostplant unknown.

\section{References}

Benson, R. B. 1968: Hymenoptera from Turkey, Symphyta. - Bulletin of the British Museum (Natural History). - Entomology series, London 22(4): 111-207.

ÇAlmaşur, Ö. \& ÖzBeK, H. 2004a: A contribution to the knowledge of the Tenthredinidae. (Symphyta, Hymenoptera) fauna of Turkey. Part I: The Subfamily Tenthredininae. - Türkish Journal of Zoology 28 : 37-54.

Çalmaşur, Ö. \& ÖzbeK, H. 2004b: A contribution to the knowledge of Tenthredinidae (Symphyta, Hymenoptera) fauna of Turkey Part II: Subfamilies Blennocampinae, Dolerinae, Nematinae and Selandrinae. - Türkish Journal of Zoology 28: 55-71.

Çalmaşur, Ö. \& ÖzbeK, H. 2006: Check-list of the Argidae fauna (Hymenoptera: Symphyta) of Turkey. Zoology of the Middle East, 39: 89-96.

ÇAlmaşur, Ö. 2006: Four new records for the Turkish Tenthredinidae. - Türkiye Entomoloji Dergisi, 30 (3): 201-207.

Chevin, H. \& Desmier de Chenon, R. 1982: Contribution à la faune des Hyménoptères Symphytes de Turquie. - Bulletin de la Société Entomologique de France, Paris 87: 43-47.

Enslin, E. 1910a: Systematische Bearbeitung der paläarktischen Arten des Tenthrediniden-Genus Macrophya Dahlb. (Hym.). - Deutsche Entomologische Zeitschrift, Berlin [1910](5): 465-503.

EnsLin, E. 1910b: Das Tenthrediniden-Genus Allantus Jur. - Russkoje entomologitscheskoje obozrenije, S. Peterburg 10(4): 335-372.

GussakovskiJ, V. V. 1935: Insectes Hyménoptères, Chalastogastra 1. - Fauna SSSR, Moskva, Leningrad 2(1): 1-453.

Gussakovskis, V. V. 1947: Insectes Hyménoptères, Chalastogastra 2. - Fauna SSSR, Moskva, Leningrad Academie des Sciences de l'URSS, Moscou, Leningrad 2(2): 1-235.

Roller, L., 1999: Check list of the sawflies (Hymenoptera: Symphyta) of Slovakia. - Entomological Problems 30(2): 37-48.

Roller, L. \& Haris, A. 2008: Sawflies of the Carpathian Basin, history and current research - Natura Somogyiensis 11: 1-259.

TAEGER, A. 1992: Fünfter Beitrag zur Systematik der Blattwespengattung Tenthredo L. (Hymenoptera, Symphyta). v Beiträge zur Entomologie 42(1): 3-53.

YILDIRIM, E. 2016: The current knowledge of some hymenopterous families (Insecta: Hymenoptera) in Turkey. - Linzer biologische Beiträge 48 (2): 1817-1822.

Zhelochovtsev, A. N. 1988: Otryad Hymenoptera - Pereponchatokrylye, Podotryad Symphyta Sidyachebryukhie, 7-234. In: Medvedev, K.H. (ed.) Opredelitel nasekomykh evropeiskoi chasti SSSR, Vol. 3 Hymenoptera, Part 6, Nauka, Leningrad. 
